\title{
Genetic diversity and dynamics of Plasmodium falciparum and $P$. vivax populations in multiply infected children with asymptomatic malaria infections in Papua New Guinea
}

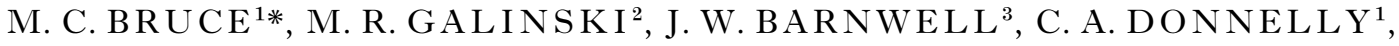 \\ M. WALMSLEY ${ }^{4}$, M. P. ALPERS ${ }^{5}$, D. WALLIKER ${ }^{4}$ and K. P. DAY ${ }^{1}$ \\ ${ }^{1}$ Wellcome Trust Centre for the Epidemiology of Infectious Disease, Department of Zoology, University of Oxford, \\ Oxford OX1 $3 F Y$ \\ ${ }^{2}$ Department of Medicine, Division of Infectious Diseases, Emory University School of Medicine, Emory Vaccine Center, \\ Atlanta, GA 30329, USA \\ ${ }^{3}$ Biology and Diagnostic Branch, Division of Parasitic Diseases, Centers for Disease Control and Prevention, Atlanta, \\ GA 30341, USA \\ ${ }^{4}$ Institute of Cell, Animal and Population Biology, University of Edinburgh, King's Buildings, Edinburgh EH9 $37 N$ \\ ${ }^{5}$ Papua New Guinea Institute of Medical Research, P.O. Box 378, Madang, Papua New Guinea
}

(Received 9 November 1999; revised 16 March 2000; accepted 16 March 2000)

SU M M ARY

We describe the dynamics of co-infections of Plasmodium falciparum and P. vivax in 28 asymptomatic children by genotyping these species using the polymorphic loci Msp2 and Msp3 $\alpha$, respectively. The total number of Plasmodium spp. infections detected using 3 day sampling over 61 days varied between 1 and 14 (mean 6.6). The dynamics of $P$. falciparum and $P$. vivax genotypes varied greatly both within and amongst children. Periodicity in the detection of $P$. falciparum infections is consistent with the synchronous replication of individual genotypes. Replication synchrony of multiple coinfecting genotypes was not detected. In 4-year-old children $P$. falciparum genotype complexity was reduced and episodes lasted significantly longer (median duration $>60$ days) when compared to children aged 5-14 years (median duration 9 days). P. vivax genotype complexity was not correlated with age but the episode duration was also longer for this species in 4-year-olds than in older children but was not as long as $P$. falciparum episodes. Recurrence of $P$. falciparum and $P$. vivax genotypes over weeks was observed. We interpret these major fluctuations in the density of genotypes over time as the result of the mechanism of antigenic variation thought to be present in these Plasmodium species.

Key words: Plasmodium falciparum, Plasmodium vivax, dynamics, asymptomatic infection, genotyping.

\section{INTRODUCTION}

Malaria parasite populations are made up of multiple genetic variants. The turnover in Plasmodium populations over short intervals of time within single human hosts has been shown to be high in regions of high endemicity, both for P. falciparum (Daubersies et al. 1996; Färnert et al. 1997) and P. vivax (Bruce et al. 1999). The rate of turnover depends upon the rate of acquisition of new infections and the rate of clearance of existing infections. Clearance rates depend on immunity that is species- and most probably genotype-specific (Ciuca, Ballif \& Chelarescu-Vieru, 1934; Taliaferro, 1939; Brown \& Brown, 1965). Evidence is accumulating that important targets of naturally acquired immunity that

* Corresponding author: Wellcome Trust Centre for the Epidemiology of Infectious Disease, Department of Zoology, University of Oxford, Oxford OX1 3FY. Tel: +01865 271247. Fax: +01865 281245. E-mail: marianbruce@hotmail.com regulates parasite clearance are molecules on the surface of infected erythrocytes which undergo antigenic variation (Brown \& Brown, 1965; Mendis, Ihalamulla \& David, 1988; Baruch et al. 1995; Reeder \& Brown, 1996; Al-Khedery, Barnwell \& Galinski, 1999). Polymorphic molecules on the surface of merozoites may also play a role in protective immunity (Riley et al. 1992; Taylor et al. 1998) but the relative importance of the response to each type of molecule is not known.

We have previously shown that most individuals living in a highly malaria-endemic region of Papua New Guinea carry multiple species infections that fluctuate over time (Bruce et al. 2000a). Both $P$. falciparum and $P$. vivax infections in this region are known to consist of multiple genotypes (Paul et al. 1995; Kolakovich et al. 1996; Bruce et al. 1999, $2000 a$ ).

However, the nature of the factors that determine the fluctuations in parasitaemia of such multiple infections are not fully understood. It has been 
suggested that innate immune responses play an important role in regulating parasite density in asymptomatic children (Kwiatkowski \& Nowak, 1991; Kwiatkowski, 1995). Non-specific regulatory mechanisms are thought to act in concert with specific responses against diverse Plasmodium antigens resulting in an interaction between co-infecting species that can alter the pattern of infection of each species (Bruce et al. 2000a).

To study the dynamics of Plasmodium infections frequent sampling from patient's blood is required at intervals close to that of the parasite replication cycle, i.e. $48 \mathrm{~h}$ for $P$. falciparum and $P$. vivax. In previous studies, dynamics of multiple clones of $P$. falciparum in asymptomatic hosts have been followed by daily sampling over short periods (Daubersies et al. 1996; Färnert et al. 1997). In this study, we have examined samples of parasitized blood from patients in Papua New Guinea every 3 days over a 61 day period. By plotting the genotype data of both $P$. falciparum and $P$. vivax with species density data we have built up an integrated picture of the dynamics of co-infections over time. For the first time, this study has allowed re-interpretation of the nature of multi-species infections normally identified only by microscopy.

\section{MATERIALS AND METHODS}

\section{Study area, population and sampling protocol}

These are described in an accompanying paper (Bruce et al. 2000b).

\section{Sample collection and DNA extraction}

Samples for genetic analysis were collected at the same time as blood smears (see Bruce et al. 2000 a, b). Approximately $500 \mu \mathrm{l}$ of blood were collected into anti-coagulent tubes containing $\mathrm{Na}_{2}$ EDTA (Sarstedt). Genotyping was carried out only on samples from children (aged 4-14 years), due to the greater prevalence of infection in children compared to adults. Children with the highest prevalence of infection were selected. P. falciparum genotyping was carried out on all samples (including those negative by microscopy) from children with 2 or more $P$. falciparum smear-positive samples. $P$. vivax genotyping was carried out on samples from children with 4 or more $P$. vivax smear-positive samples. Samples from 1 child aged 4 years with only $2 P$. vivax smear positive samples were also analysed, to allow better comparison across age groups. Samples were stored at $-70{ }^{\circ} \mathrm{C}$ before transfer of $20 \mu 1$ of blood to filter paper (Whatman no.3). DNA extraction was carried out using a Chelex boiling method (Kyes et al. 1993) and the final extract volume was $200 \mu 1$.

\section{P. falciparum genotyping}

Genetic analysis of $P$. falciparum populations was carried out for detection of allelic diversity at the $P$. falciparum merozoite surface protein 2 (Msp2) locus (Fenton et al. 1991; Smythe et al. 1991). Nested polymerase chain reaction (PCR) amplification of alleles was carried out using $2 \mu \mathrm{l}$ of DNA extract in the primary reaction and $2-0.02 \mu \mathrm{l}$ of the primary reaction as target in the nested reaction. PCR reaction volumes were $20 \mu \mathrm{l}$. Deoxynucleotide triphosphates were at $0.75 \mathrm{~mm}$, oligonucleotide primers at $0 \cdot 1 \mu \mathrm{M}$ and $0 \cdot 5$ units of Taq polymerase (Promega) were used per reaction. Reaction buffer was as supplied, with the addition of $2.5 \mathrm{~mm} \mathrm{MgCl}_{2}$. Primer sequences and cycling temperatures were as previously described (Ranford-Cartwright et al. 1993). Amplification products were run on $1.8 \%$ agarose gels stained with ethidium bromide. Alleles were discriminated by both product size and sequence dimorphism (Smythe et al. 1991). Size was determined to within $20 \mathrm{bp}$ using a standard curve drawn from DNA size standards (BoehringerMannheim). Sequence type was determined by Southern blotting of products to nylon membrane and hybridization with IC1-type and FC27-type sequence-specific probes, followed by chemiluminescent detection (Amersham) (Babiker et al. 1994).

\section{P. vivax genotyping}

P. vivax genotypes were distinguished using PCR/ restriction fragment length polymorphism (RFLP) analysis (Bruce et al. 1999) of alleles at the merozoite surface protein 3 alpha (Msp3 $\alpha$ ) locus (Galinski et al. 1999). The combination of RFLPs from HhaI and AluI digests were used to differentiate genotypes (Bruce et al. 1999). Multiple $P$. vivax populations present in a single sample were easily distinguished as they resulted in multiple PCR products or RFLP patterns in which fragment sizes sum to greater than that of the uncut product. A consensus approach was taken in assigning genotypes in samples from individual children containing mixed parasite populations. Where a mixed pattern contained fragment sizes identical to those observed in one or more other samples from the same child these patterns were assigned. Only when the observed fragment sizes could not be a combination of other patterns observed within samples from a child was a new genotype assumed.

\section{Genotyping replicates, controls and sensitivity}

One negative control was run per 10 samples in all primary and nested reactions. Samples in which multiple products were observed, were repeated 
with $1 / 100$ th the amount of primary reaction transferred to the nested to ensure that these were not a result of carry-over. Where replicate results varied due to stochasticity in the amplification of low density populations, genotypes observed in both amplifications were used. Samples from individual children were run in chronological order on gels. Genotype identity between children was ensured by running representative samples containing each genotype from each child on the same gel. Replicates were carried out for $65 \%$ of smear-positive and $77 \%$ of smear-negative $P$. falciparum samples genotyped. Eighty-five per cent of $P$. vivax smear-positive samples were assayed in replicate and due to the lower sensitivity of $P$. vivax genotyping of a selection of $20 \%$ of smear-negative samples covering all children, was duplicated.

The lower limit of the sensitivity of detection of $P$. falciparum genotypes was tested using mock field samples containing $1000-0.5$ parasites/ $\mu 1$ in $40 \%$ haematocrit blood. In vitro-cultured parasite clones of different allelic types (3D7 and Ro33, ICI-type and HB3, FC27-type) were combined to assess sensitivity of detection and allelic amplification bias in mixed infections with varying ratios. The lower sensitivity limit of $P$. vivax genotyping was previously determined as 100 parasites/ $\mu 1$ (Bruce et al. 1999).

\section{Statistical analysis}

To test for periodicity in the detection of individual $P$. falciparum and $P$. vivax genotypes a paired sample analysis was carried out to calculate the conditional probability of infection with the same genotype at 3-60 day intervals following detection in the first of the pair of samples. This was carried out as for blood smear data (Bruce et al. 2000b).

Duration of episodes of infection with individual genotypes of $P$. falciparum and $P$. vivax was measured according to the same rules as used for microscopy data (Bruce et al. 2000b). Episodes were defined as consecutive genotype positive samples for $P$. vivax. For $P$. falciparum, genotype episodes were taken as consecutive or 6 day periodic positive time points to allow for observed periodicity in detection of genotypes of this species (see Results section). Episodes defined using consecutive or 6 day periodic samples are called moderate estimates. A maximum measure of duration was also taken. This was the time between the first and the last positive sample for each genotype within each child. Survival analysis of genotype duration estimates was carried out. Episodes estimated using the moderate method were considered censored if they bounded either end of the study period. Maximum estimates were censored if they fell within 2 days of the beginning or end of the study period.

\section{RESULTS}

The genetic identity of $P$. falciparum and $P$. vivax infections were determined from longitudinal samples from 28 children aged 4-14 years, living in a malaria endemic region of Papua New Guinea. $P$. falciparum genotypes were determined in samples from $25 / 28$ children, $P$. vivax genotypes were determined for $16 / 28$ children and $13 / 28$ children were analysed for both species (Table 1). Genotype data for all children can be obtained at http:// www.ceid.ox.ac.uk/download/. For both species, single loci were used for genetic typing and we refer to this as the parasite 'genotype' throughout. Multilocus typing was precluded due to the frequent presence of multiple infections. Alleles at the Msp2 locus of $P$. falciparum and those at the Msp $3 \alpha$ locus of $P$. vivax were used to distinguish individual infections of these species.

\section{Genetic diversity at the Msp2 locus of $\mathrm{P}$. falciparum}

A total of 395 samples were available from 25 children selected for $P$. falciparum genotyping (Table 1). Genotypes could be assigned in 227/395 samples $(57 \cdot 5 \%)$. P. falciparum parasites were detected by microscopy in only $62.1 \%$ of samples positive by PCR (Table 1), illustrating the higher sensitivity of PCR detection. A total of $305 / 395$ $(77 \cdot 2 \%)$ samples were assayed in duplicate. Replicates of 150 samples were consistently negative ( $87.7 \%$ of these were $P$. falciparum smear-negative) and in 41 samples amplification occurred in only 1 replicate. In 114 samples amplification was observed in both replicates and in $72.8 \%$ of these samples, results were identical. In the remainder, a difference in the number and/or size of products was observed. Such differences have been commonly observed by other workers (Molecular Epidemiology in Malaria Collaborative Research Network (Björkman et al. 1998, personal communication) and are likely to be a result of random amplification of populations present at densities close to the detection threshold, estimated to be approximately 10 parasites $/ \mu 1$ of blood in this study. A total of 27 distinct Msp2 alleles were found in 227 PCR-positive samples (Fig. 1 A and Table 2). Eight null alleles did not react with either repeat-specific probe.

\section{Genetic diversity at the $M s p 3 \alpha$ locus of $\mathrm{P}$. vivax}

A total of 258 samples were available for PCR analysis from 16 children selected for $P$. vivax genotyping (Table 1). PCR amplification at the Msp3 $\alpha$ locus was successful in 95/258 (36.8\%) samples, of which 57 were smear-positive. Of these, $108 / 258$ samples $(41.9 \%)$ were assayed in duplicate. Replicates of 64 samples were consistently negative 
Table 1. Number of samples used in PCR genotyping and genotypes detected per child for Plasmodium falciparum and P. vivax

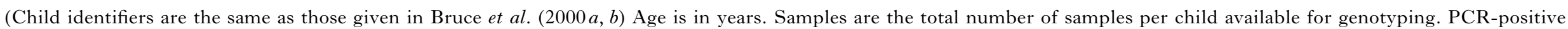

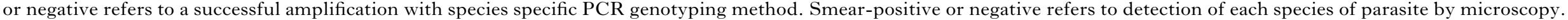

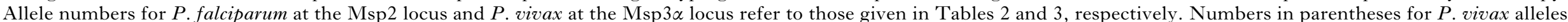

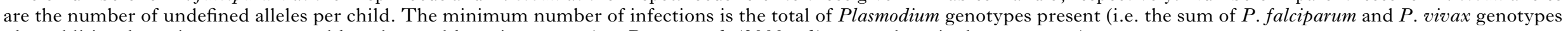
plus additional species not genotyped but detected by microscopy (see Bruce et al. $(2000 a, b)$ counted as single genotypes.)

\begin{tabular}{|c|c|c|c|c|c|c|c|c|c|c|c|c|c|c|c|}
\hline Child & Sex & Age & Samples & $\begin{array}{l}\text { PCR } \\
+ \text { ve } \\
\text { smear } \\
+ \text { ve }\end{array}$ & $\begin{array}{l}\text { PCR } \\
+ \text { ve } \\
\text { smear } \\
\text {-ve }\end{array}$ & $\begin{array}{l}\text { PCR } \\
\text { - ve } \\
\text { smear } \\
+ \text { ve }\end{array}$ & $\begin{array}{l}\text { Geno- } \\
\text { types/ } \\
\text { child }\end{array}$ & $\begin{array}{l}\text { Msp2 alleles } \\
\text { detected }\end{array}$ & Samples & $\begin{array}{l}\text { PCR } \\
+ \text { ve } \\
\text { smear } \\
+ \text { ve }\end{array}$ & $\begin{array}{l}\text { PCR } \\
+ \text { ve } \\
\text { smear } \\
- \text { ve }\end{array}$ & $\begin{array}{l}\text { PCR } \\
- \text { ve } \\
\text { smear } \\
+ \text { ve }\end{array}$ & $\begin{array}{l}\text { Geno- } \\
\text { types/ } \\
\text { child }\end{array}$ & $\begin{array}{l}\text { Msp3 } \alpha \text { alleles } \\
\text { detected }\end{array}$ & $\begin{array}{l}\text { Minimum } \\
\text { number of } \\
\text { infections }\end{array}$ \\
\hline 1 & $\mathrm{~m}$ & 4 & 14 & 7 & 6 & 0 & 1 & 9 & 14 & 7 & 0 & 1 & 4 & $1,18(2)$ & 5 \\
\hline 2 & $\mathrm{~m}$ & 4 & 18 & 8 & 5 & 1 & 1 & 16 & N.D. & N.D. & N.D. & N.D. & N.D. & N.D. & 1 \\
\hline 4 & $\mathrm{f}$ & 4 & 12 & 6 & 2 & 1 & 1 & 27 & 12 & 2 & 1 & 0 & 1 & 15 & 2 \\
\hline 5 & $\mathrm{~m}$ & 4 & 16 & 13 & 2 & 1 & 2 & 3,25 & 16 & 7 & 4 & 3 & 7 & $1,7,9(4)$ & 9 \\
\hline 6 & $\mathrm{~m}$ & 7 & 11 & 7 & 4 & 0 & 6 & $3,4,5,10,14,17$ & N.D. & N.D. & N.D. & N.D. & N.D. & N.D. & 8 \\
\hline 7 & $\mathrm{~m}$ & 7 & 17 & 4 & 4 & 1 & 3 & $6,9,16$ & N.D. & N.D. & N.D. & N.D. & N.D. & N.D. & 5 \\
\hline 8 & $\mathrm{f}$ & 7 & 19 & 2 & 4 & 0 & 3 & $1,3,9$ & 19 & 2 & 1 & 3 & 1 & 17 & 4 \\
\hline 10 & $\mathrm{~m}$ & 8 & 16 & 3 & 6 & 0 & 5 & $9,12,14,18,24$ & 16 & 3 & 4 & 2 & 3 & $7,16(1)$ & 8 \\
\hline 11 & $\mathrm{f}$ & 9 & 13 & 4 & 2 & 0 & 5 & $6,7,9,10,14$ & N.D. & N.D. & N.D. & N.D. & N.D. & N.D. & 6 \\
\hline 12 & $\mathrm{~m}$ & 8 & 17 & 8 & 3 & 2 & 5 & $3,4,9,13,15$ & 17 & 1 & 0 & 2 & 1 & 1 & 7 \\
\hline 14 & $\mathrm{f}$ & 6 & N.D. & N.D. & N.D. & N.D. & N.D. & N.D. & 19 & 8 & 1 & 3 & 3 & $16,22(1)$ & 4 \\
\hline 15 & $\mathrm{f}$ & 9 & N.D. & N.D. & N.D. & N.D. & N.D. & N.D. & 19 & 4 & 7 & 2 & 4 & $1,10(2)$ & 6 \\
\hline 16 & $\mathrm{f}$ & 7 & 15 & 7 & 2 & 1 & 6 & $4,8,15,18,19,21$ & 14 & 7 & 1 & 1 & 6 & $1,6,11(3)$ & 14 \\
\hline 17 & $\mathrm{~m}$ & 6 & 18 & 6 & 1 & 1 & 4 & $4,13,15,24$ & N.D. & N.D. & N.D. & N.D. & N.D. & N.D. & 6 \\
\hline 18 & $\mathrm{f}$ & 5 & 13 & 4 & 7 & 1 & 4 & $3,9,11,14$ & 13 & 1 & 2 & 2 & 3 & $6,5,23$ & 7 \\
\hline 19 & $\mathrm{~m}$ & 14 & 17 & 7 & 3 & 0 & 5 & $3,5,6,11,15$ & N.D. & N.D. & N.D. & N.D. & N.D. & N.D. & 7 \\
\hline 20 & $\mathrm{~m}$ & 14 & 15 & 3 & 6 & 2 & 6 & $4,7,11,12,15,16$ & 14 & 2 & 0 & 3 & 3 & $3(2)$ & 10 \\
\hline 21 & $\mathrm{f}$ & 11 & 17 & 4 & 6 & 0 & 2 & 5,10 & N.D. & N.D. & N.D. & N.D. & N.D. & N.D. & 2 \\
\hline 22 & $\mathrm{f}$ & 14 & 18 & 6 & 3 & 3 & 4 & $4,14,16,18$ & 18 & 2 & 3 & 2 & 3 & $24(2)$ & 8 \\
\hline 24 & $\mathrm{~m}$ & 14 & 17 & 0 & 1 & 3 & 1 & 5 & 17 & 3 & 4 & 5 & 8 & $4,6,12,13,21(3)$ & 10 \\
\hline 25 & $\mathrm{~m}$ & 12 & 13 & 4 & 3 & 0 & 5 & $3,14,17,25,26$ & N.D. & N.D. & N.D. & N.D. & N.D. & N.D. & 7 \\
\hline 26 & $\mathrm{~m}$ & 12 & 17 & 4 & 4 & 0 & 4 & $9,11,12,23$ & N.D. & N.D. & N.D. & N.D. & N.D. & N.D. & 6 \\
\hline 27 & $\mathrm{f}$ & 10 & N.D. & N.D. & N.D. & N.D. & N.D. & N.D. & 19 & 2 & 5 & 3 & 4 & $1,9,19,20$ & 5 \\
\hline 28 & $\mathrm{f}$ & 11 & 18 & 1 & 0 & 1 & 1 & 16 & N.D. & N.D. & N.D. & N.D. & N.D. & N.D. & 2 \\
\hline 31 & $\mathrm{~m}$ & 10 & 16 & 11 & 3 & 1 & 9 & $3,4,9,10,11,12,13,14,15$ & 16 & 1 & 3 & 3 & 4 & $2,14(2)$ & 13 \\
\hline 32 & $\mathrm{~m}$ & 13 & 19 & 10 & 5 & 2 & 5 & $2,5,15,16,22$ & N.D. & N.D. & N.D. & N.D. & N.D. & N.D. & 6 \\
\hline 33 & $\mathrm{f}$ & 10 & 15 & 5 & 3 & 0 & 3 & $3,4,5$ & 15 & 5 & 2 & 5 & 7 & $2,8(5)$ & 10 \\
\hline 34 & $\mathrm{~m}$ & 14 & 14 & 7 & 1 & 2 & 5 & $2,4,13,15,20$ & N.D. & N.D. & N.D. & N.D. & N.D. & N.D. & 7 \\
\hline Total & & & 395 & 141 & 86 & 23 & & & 258 & 57 & 38 & 40 & & & \\
\hline
\end{tabular}

N.D., Not determined. 


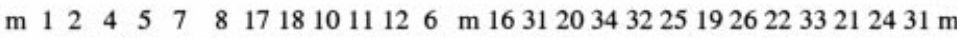

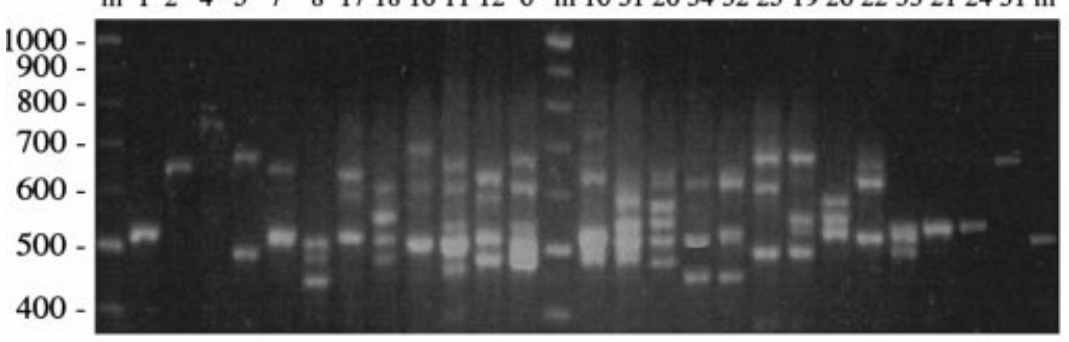

B
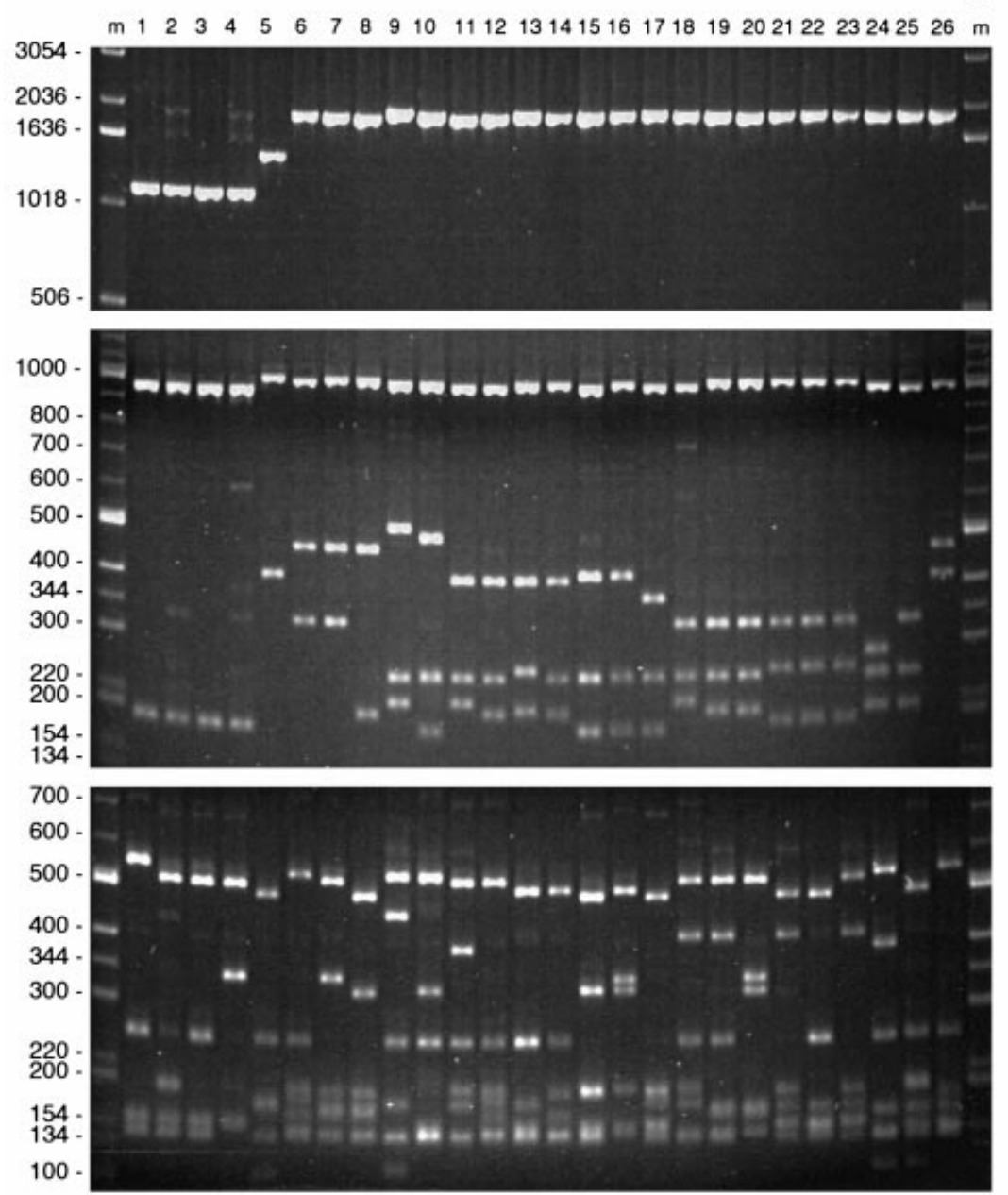

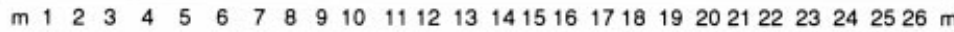

Fig. 1. Allelic polymorphism at (A) the Plasmodium falciparum Msp2 locus and (B) the P. vivax Msp3 $\alpha$ locus. Msp2 alleles from multiple time-points during the 61 day study period have been combined to show the size polymorphism present within each child during the study. Lanes in (A) are numbered with the child identifiers as in Table 1. Due to the higher background when samples are pooled some alleles are detectable only as faint bands, and some, which are close in size, appear merged. Msp2 allele 13 in child 34 is not shown. Msp3 $\alpha$ alleles are distinguished by size (top panel) and RFLP patterns after digestion with restriction enzymes HhaI (middle panel) and AluI (bottom panel). Lanes 1-24 are labelled with the allele number as referred to in Table 1 and 3. Lanes 25 and 26 contain products from $P$. vivax laboratory strains Belem and Sal 1, respectively. Sizes of markers, Lanes $\mathrm{m}$, are shown in base pairs.

$(37 \cdot 5 \%$ of these samples were $P$. vivax smearnegative) and in 18 samples amplification was observed in only 1 replicate. In 26 samples, amplification was observed in both replicates and in 16/26 identical results were obtained.

Three sizes of PCR amplification products (1100, 1400 and $1900 \mathrm{bp}$ ) were observed at the Msp3 $\alpha$ locus
(Fig. 1B, top panel and Table 3). Two of these (1100 and $1900 \mathrm{bp}$ ) had been previously described in a set of samples from Gonoa village, which contained some overlap with those presented here (Bruce et al. 1999). An increased sensitivity in distinguishing different $P$. vivax infections using a combination of $H h a \mathrm{I}$ and $A l u \mathrm{I}$ has been previously shown (Bruce $e t$ 
Table 2. Size and sequence types of alleles detected at the Plasmodium falciparum Msp2 locus

(Numbers of samples and numbers of children do not add to the total numbers assayed as detection of multiple alleles per child and per sample was common. Numbers are given for the 3 sequence categories without reference to allele size.)

\begin{tabular}{|c|c|c|c|c|}
\hline Allele & Size (bp) & $\begin{array}{l}\text { Sequence } \\
\text { type }\end{array}$ & $\begin{array}{l}\text { Number of samples in } \\
\text { which allele detected }\end{array}$ & $\begin{array}{l}\text { Number of children in } \\
\text { which allele detected }\end{array}$ \\
\hline 1 & $440-459$ & $\mathrm{FC} 27$ & 3 & 1 \\
\hline 2 & $460-479$ & $\mathrm{FC} 27$ & 7 & 2 \\
\hline 3 & $480-499$ & $\mathrm{FC} 27$ & 29 & 9 \\
\hline 4 & $500-519$ & $\mathrm{FC} 27$ & 40 & 9 \\
\hline 5 & $520-539$ & $\mathrm{FC} 27$ & 21 & 7 \\
\hline 6 & $660-679$ & IC1 & 10 & 2 \\
\hline 7 & $460-479$ & IC1 & 2 & 2 \\
\hline 8 & $480-499$ & IC1 & 1 & 1 \\
\hline 9 & $500-519$ & IC1 & 35 & 9 \\
\hline 10 & $520-539$ & IC1 & 16 & 4 \\
\hline 11 & $540-559$ & $\mathrm{IC} 1$ & 12 & 5 \\
\hline 12 & $560-579$ & IC1 & 9 & 4 \\
\hline 13 & $580-599$ & IC1 & 14 & 4 \\
\hline 14 & $600-619$ & $\mathrm{IC} 1$ & 24 & 7 \\
\hline 15 & $620-639$ & IC1 & 26 & 8 \\
\hline 16 & $640-659$ & IC1 & 27 & 6 \\
\hline 17 & $660-679$ & IC1 & 5 & 2 \\
\hline 18 & $680-699$ & $\mathrm{IC} 1$ & 6 & 3 \\
\hline 19 & $720-739$ & IC1 & 2 & 1 \\
\hline 20 & $240-259$ & Null & 1 & 1 \\
\hline 21 & $500-519$ & Null & 3 & 1 \\
\hline 22 & $520-539$ & Null & 1 & 1 \\
\hline 23 & $560-579$ & Null & 1 & 1 \\
\hline 24 & $620-639$ & Null & 4 & 2 \\
\hline 25 & $660-679$ & Null & 17 & 2 \\
\hline 26 & $700-719$ & Null & 1 & 1 \\
\hline \multirow[t]{4}{*}{27} & $740-759$ & Null & 8 & 1 \\
\hline & Any & $\mathrm{FC} 27$ & 100 & 19 \\
\hline & Any & IC1 & 144 & 21 \\
\hline & Any & Null & 36 & 9 \\
\hline
\end{tabular}

al. 1999). The combination of these RFLP patterns allowed $24 \mathrm{Msp} 3 \alpha$ alleles to be distinguished (Fig. $1 \mathrm{~B}$, and Table 3 ).

Some RFLP patterns were impossible to define as they were observed only in samples with mixed populations. Undefined genotypes were detected in 29 samples from 11 children. Despite not being able to assign restriction fragments for such genotypes these samples were still useful. Undefined genotypes could be distinguished from those present at other time-points within the same child if fragment sizes did not match. Undefined allele results were used in genotype dynamics and duration analyses as they rely only on comparison within a child and not between children.

Multiplicity of $\mathrm{P}$. falciparum and $\mathrm{P}$. vivax infections per sample

The mean multiplicity of $P$. falciparum infections across PCR-positive samples was 1.4 genotypes per sample (range $1-4)$. The number of $P$. falciparum genotypes per sample was not correlated with $P$. falciparum parasite density $(r=0.07, P=0 \cdot 287)$.
Mean multiplicity of $P$. vivax infection was 1.4 genotypes per sample (range 1-6) and there was also a lack of correlation with $P$. vivax density $(r=0 \cdot 18$, $P=0 \cdot 08)$.

\section{Multiplicity of Plasmodium spp. infections per child}

Most children were infected with multiple genotypes of each species over the course of the study (Table 1). Single genotypes were found in only $5 / 25$ children assayed for $P$. falciparum and $3 / 16$ children assayed for $P$. vivax. Up to $9 P$. falciparum genotypes (mean $=3.8)$ and 8 P. vivax genotypes $($ mean $=3 \cdot 9)$ were distinguished per child. These results have been in part reported and discussed elsewhere (Bruce et al. $2000 a$ ).

Genotypes for each species ( $P$. falciparum and $P$. vivax) were considered together with microscopy data for species not genotyped to calculate the minimum number of Plasmodium infections for each child during the study (Table 1 ). Only 1 child had a single Plasmodium infection throughout days 0-60 of the study. All others had between 2 and 14 different infections $($ mean $=6 \cdot 6)$. The number of $P$. 
Table 3. Size and RFLP patterns of alleles detected at the Plasmodium vivax Msp3 $\alpha$ locus

(The number of samples and number of children do not add to the total number assayed as detection of multiple alleles per child and per sample was common. Data for individual alleles 1-24 comes only from samples in which defined alleles were detected. Numbers are given for the 3 size categories without reference to RFLP patterns. These data are from all samples in which PCR amplification was successful. AluI RFLP pattern a10 is not used to define any allele as it was only detected in samples shown to be mixed infections using HhaI digestion. HhaI patterns h1, h3, h8, h9, h12, h13 and AluI pattern a11 are used, in combination with other patterns to define multiple alleles.)

\begin{tabular}{|c|c|c|c|c|c|}
\hline Allele & $\begin{array}{l}\text { Size } \\
\text { (bp) }\end{array}$ & $\begin{array}{l}\text { HhaI RFLP } \\
\text { pattern }\end{array}$ & $\begin{array}{l}\text { AluI RFLP } \\
\text { pattern }\end{array}$ & $\begin{array}{l}\text { Number of samples in } \\
\text { which allele detected }\end{array}$ & $\begin{array}{l}\text { Number of children in } \\
\text { which allele detected }\end{array}$ \\
\hline 1 & 1100 & h1 & a1 & 20 & 6 \\
\hline 2 & 1100 & h1 & $\mathrm{a} 2$ & 2 & 2 \\
\hline 3 & 1100 & h1 & a3 & 2 & 1 \\
\hline 4 & 1100 & h1 & $\mathrm{a} 4$ & 1 & 1 \\
\hline 5 & 1400 & h2 & a6 & 1 & 1 \\
\hline 6 & 1900 & h3 & a11 & 5 & 3 \\
\hline 7 & 1900 & h3 & a18 & 6 & 2 \\
\hline 8 & 1900 & h4 & a19 & 3 & 1 \\
\hline 9 & 1900 & h5 & a12 & 8 & 2 \\
\hline 10 & 1900 & h6 & $\mathrm{a} 21$ & 4 & 1 \\
\hline 11 & 1900 & h7 & a17 & 3 & 1 \\
\hline 12 & 1900 & h8 & a11 & 1 & 1 \\
\hline 13 & 1900 & h8 & $\mathrm{a} 8$ & 1 & 1 \\
\hline 14 & 1900 & h8 & a9 & 1 & 1 \\
\hline 15 & 1900 & h9 & a20 & 3 & 1 \\
\hline 16 & 1900 & h9 & a22 & 10 & 2 \\
\hline 17 & 1900 & h10 & a5 & 3 & 1 \\
\hline 18 & 1900 & h11 & a14 & 1 & 1 \\
\hline 19 & 1900 & h12 & a13 & 1 & 1 \\
\hline 20 & 1900 & h12 & $\mathrm{a} 23$ & 2 & 1 \\
\hline 21 & 1900 & h13 & a16 & 1 & 1 \\
\hline 22 & 1900 & h13 & a7 & 2 & 1 \\
\hline 23 & 1900 & h13 & a24 & 1 & 1 \\
\hline \multirow[t]{4}{*}{24} & 1900 & h14 & a15 & 5 & 1 \\
\hline & 1100 & & & 24 & 10 \\
\hline & 1400 & & & 7 & 3 \\
\hline & 1900 & & & 74 & 15 \\
\hline
\end{tabular}

falciparum genotypes was not correlated with that for $P$. vivax amongst the 13 children whose samples were genotyped for both species $(r=-0 \cdot 163, P=$ $0 \cdot 594)$. The number of genotypes present per child was not correlated with age for either $P$. falciparum or $P$. vivax when all children were analysed together (P. falciparum: $r=0.316, P=0.312 ; P$. vivax $: r=$ $0 \cdot 219, P=0 \cdot 413$ ) nor was the total number of all Plasmodium infections, when all 4 species were considered together (Table 1$)(r=0 \cdot 262, P=0 \cdot 178)$. Despite the lack of correlation with age there was a notable reduction in the complexity of $P$. falciparum infections in 4-year-old children compared to older children. Three out of 4 of these children have a single $P$.falciparum infection during the whole study period and the other had only 2 infections.

A correlation between the proportion of timepoints microscopy positive for $P$. vivax and the number of $P$. vivax genotypes has been previously reported (Bruce et al. $2000 a$ ). This relationship also holds for $P$. falciparum in children aged 5-14 years but not $P$. falciparum for 4-year-olds (Bruce et al. $2000 a$ ). In addition, the total number of Plasmodium infections (Table 1) shows a significant association with the number of smear-positive samples of any species in 5 to 14 -year-olds $(r=0.453, P=0.026)$ but when 4 -year-old children were also included significance was abolished $(r=0 \cdot 330, P=0 \cdot 086)$.

\section{Dynamics of PCR-detectable P. falciparum genotypes}

The dynamics of PCR-detectable $P$. falciparum infections differed greatly both within and between individual children. The simplest dynamics were observed in P. falciparum infections in children aged 4 years (Fig. 2, child 1). In all of the 4-year-old children a single $P$. falciparum infection lasted for almost the total duration of the study.

Dynamics of P. falciparum infections were significantly different in children greater than 4 years of age. The pattern of infection was more complex in 5 to 14-year-olds (Fig. 2, children 22, 6 and 31). The dynamics of individual genotypes were characterized by shorter periods of infection and some were seen on only a single occasion per child. Some $P$. 


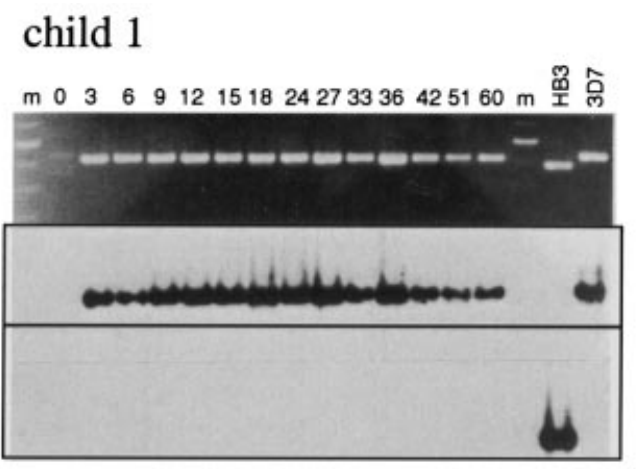

\section{child 22}
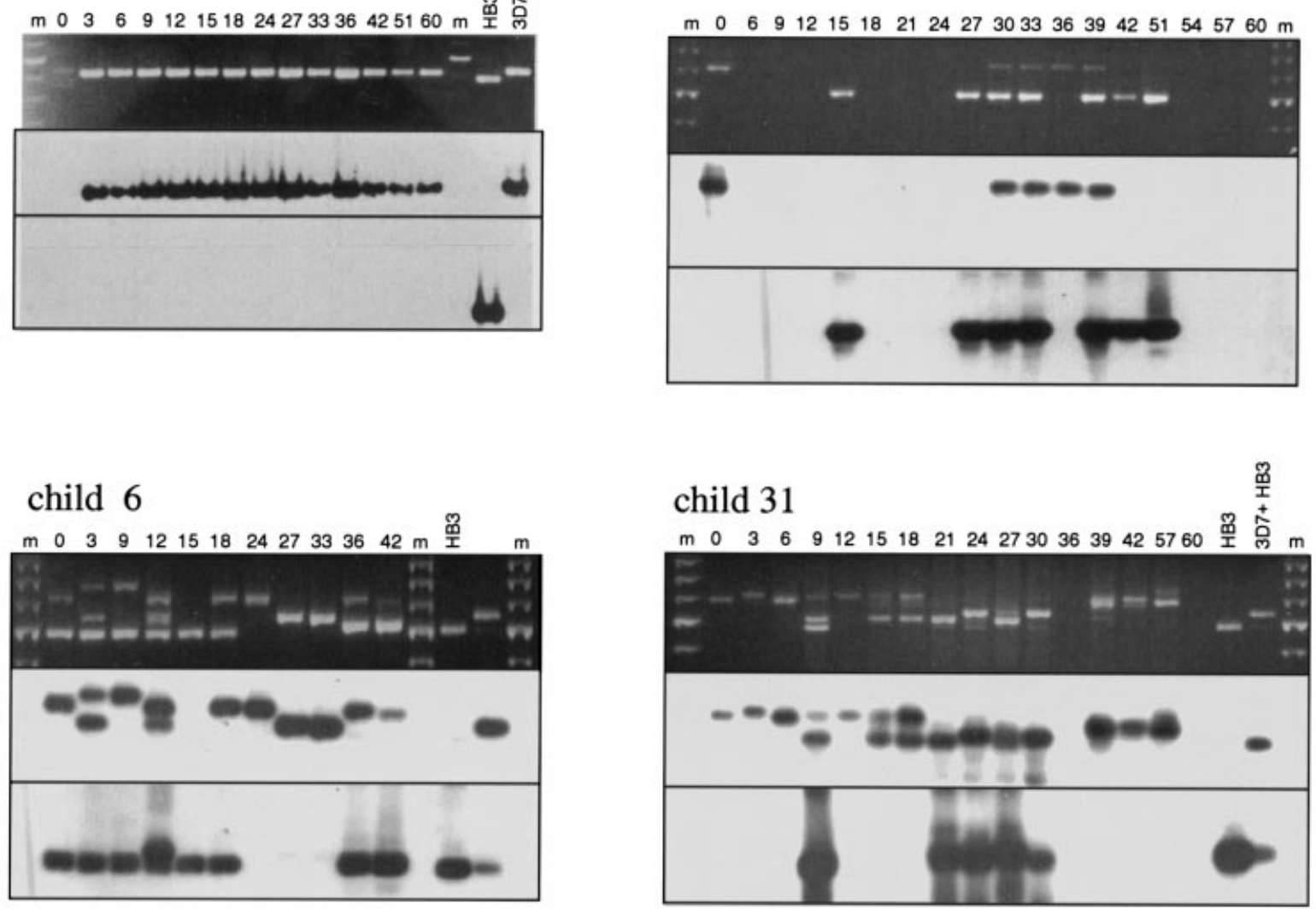

Fig. 2. Intra-host dynamics of Plasmodium falciparum genotypes in 4 children detected by allelic variation at the Msp2 locus. Data available from time-points throughout the study period, including those negative for PCR amplification products, are shown. The child identity number (Table 1) is shown at the top of each set of samples. The top panels show size polymorphism of PCR products. Lower panels show sequence dimorphism of products detected by hybridization of products with sequence-specific oligonucleotide probes: IC1 type (middle panels), FC27 (lower panels). DNA size marker fragment with double intensity in Lanes $\mathrm{m}$, is $500 \mathrm{bp}$. Fragments above and below have increments or decrements of $100 \mathrm{bp}$. Products amplified from laboratory lines 3D7 and HB3 shown in some panels are hybridization controls for IC1 and FC27-type probes, respectively.

falciparum infections were seen at consecutive timepoints (e.g. Fig. 2, child 22 and child 6) but others were characterized by periodic detection at 6 day intervals. This periodicity was investigated statistically (see below). Recurrence of genotypes also occurred at longer intervals e.g. in child 22 (Fig. 2), the $\mathrm{FC} 27$ genotype was detected on day 15 but not again until days $27-33$. The appearance and clearance of individual genotypes was independent of other co-infecting $P$. falciparum genotypes.

\section{Dynamics of PCR-detectable P. vivax genotypes}

The dynamics of $P$. vivax infections were also variable within and between individuals (Fig. 3). The same genotype was detected at sequential timepoints in some children (e.g. Fig. 3, child 4) and in others detection was more sporadic. Some genotypes were only detected at a single time-point (e.g. Fig. 3, child 18). In contrast to P. falciparum, there was no difference in the dynamics of $P$. vivax infections in children aged 4 years compared to those in older children. There was apparent replacement of one genotype with another in 1 child (Fig. 3, child 10) but in most children turnover of each genotype appeared independent of co-infecting $P$. vivax infections.

Periodicity in the detection of $\mathrm{P}$. falciparum and $\mathrm{P}$. vivax genotypes

Periodicity in the detection of $P$. falciparum parasites by blood smear had been previously noted in these children (Bruce et al. 2000 b). To test if there was a corresponding periodicity in the detection of individual genotypes of $P$. falciparum, a paired analysis similar to that carried out on blood smear data was done. The conditional probability of infection with each distinct genotype after its detection in the first pair of samples from individual children was calculated for intervals of 3-60 days. A marked periodicity in the probability of infection was seen for P. falciparum (Fig. 4A). There was a statistically significant lower probability of infection at $3,9,15$, 

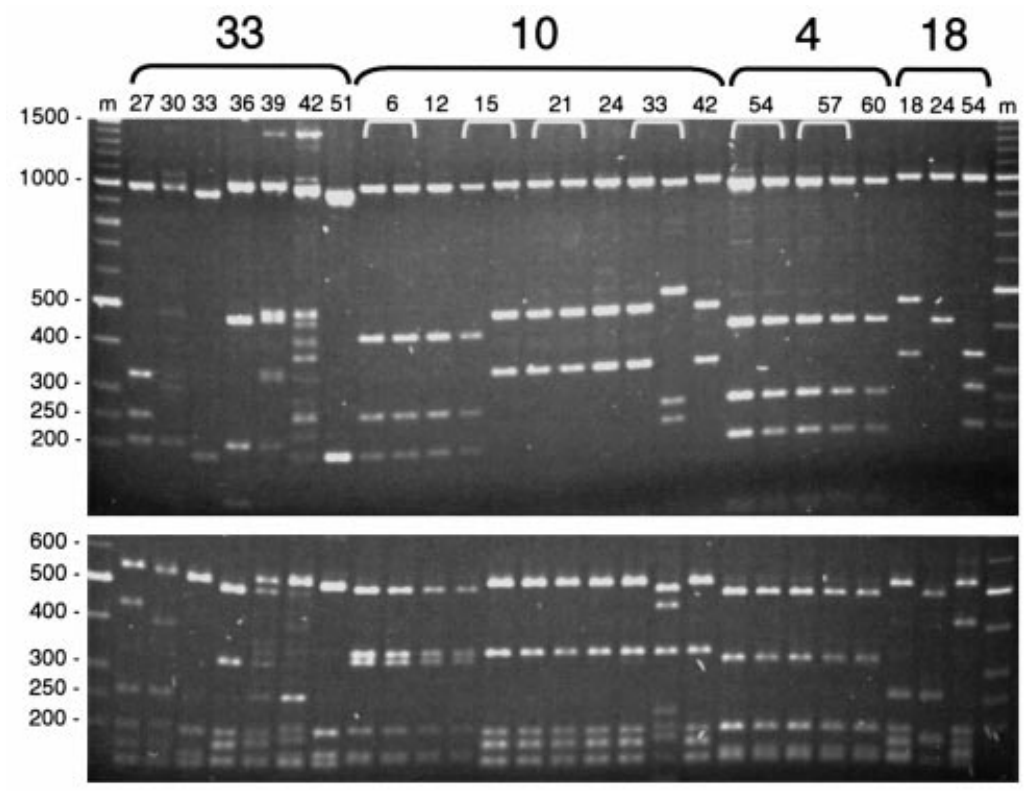

Fig. 3. Intra-host Plasmodium vivax genotype dynamics in 4 children detected by PCR amplification and RFLP analysis of allelic variation at the Msp3 $\alpha$ locus. PCR products were digested with restriction enzyme HhaI (top panel) or AluI (lower panel). The child identity number (Table 1) is shown above each set of samples bracketed in black. Samples for each child are ordered chronologically and are labelled with the time-point of collection during the study. Only samples that gave PCR products are shown. Replicate results from a single sample are shown in white brackets. Sizes of markers, Lanes $\mathrm{m}$, are shown in $\mathrm{bp}$.
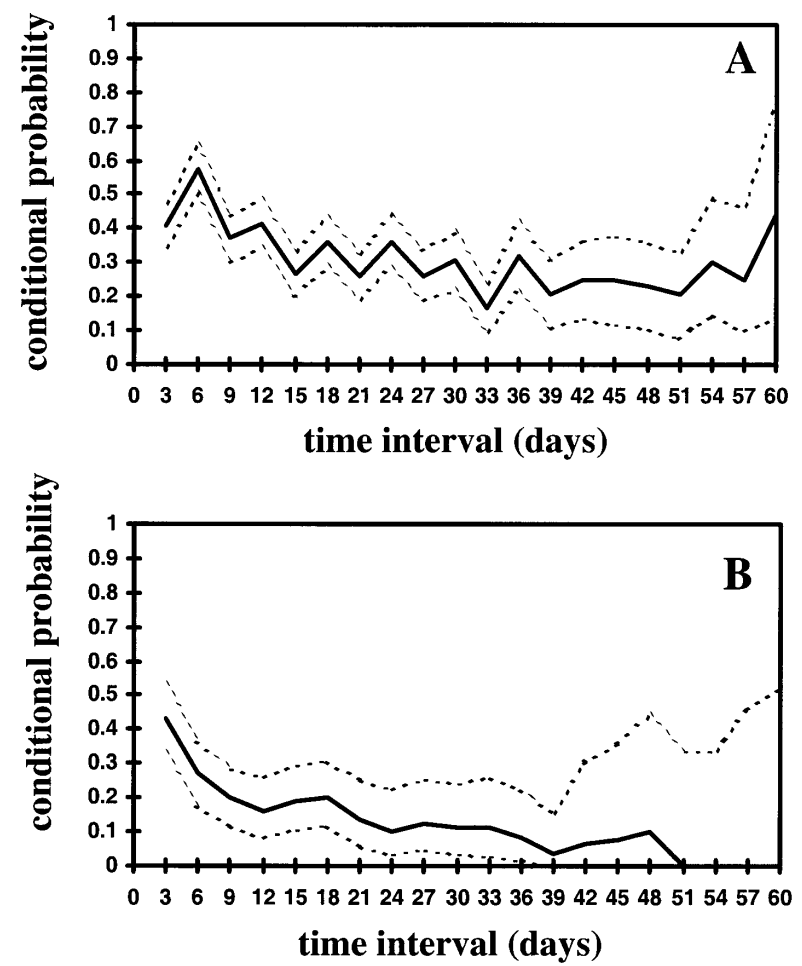

Fig. 4. Conditional probability of detection of the same genotype in pairs of samples from each individual at 3-60 day intervals for (A) Plasmodium falciparum and (B) P. vivax genotypes from children aged 4-14 years. Probability is conditional on detection of an identical genotype in the chronologically first sample in the pair.
21 and 27 day intervals than at 6,12,18 and 24 days $(P<0.05)$. Examination of the pattern of multiple $P$. falciparum genotypes in each child revealed no evidence for synchrony of different co-infecting genotypes. Conditional probabilities were also calculated for $P$. vivax genotypes for comparison but periodicity was absent from these data (Fig. 4B).

\section{Duration of genotype episodes of infection}

Estimates of the duration of genotype infections of $P$. falciparum and $P$. vivax were made using 2 different methods. Moderate estimates used the duration of consecutive positive samples for each $P$. vivax genotype. Due to periodicity in the detection of $P$. falciparum genotypes, consecutive or 6 day periodic genotype samples were used for this species. A maximum estimate was also used which measures the total detected duration of each genotype. Data for genotypes of each species from each individual were pooled by age group. The distribution of duration estimates was not significantly different between age groups 5-9 and 10-14 years for either species or method and so were combined $(P$. falciparum moderate, $\chi^{2}=0 \cdot 7,1$ D.F., $P=0 \cdot 399 ; P$. falciparum maximum, $\chi^{2}=0 \cdot 1,1$ D.F., $P=0 \cdot 770 ; P$. vivax moderate, $\chi^{2}=1 \cdot 2,1$ D.F., $P=0.557 ; P$. vivax maximum, $\chi^{2}=1 \cdot 6,1$ D.F., $P=0 \cdot 209$ ). Estimates for $P$. falciparum and $P$. vivax for children aged 4 years 

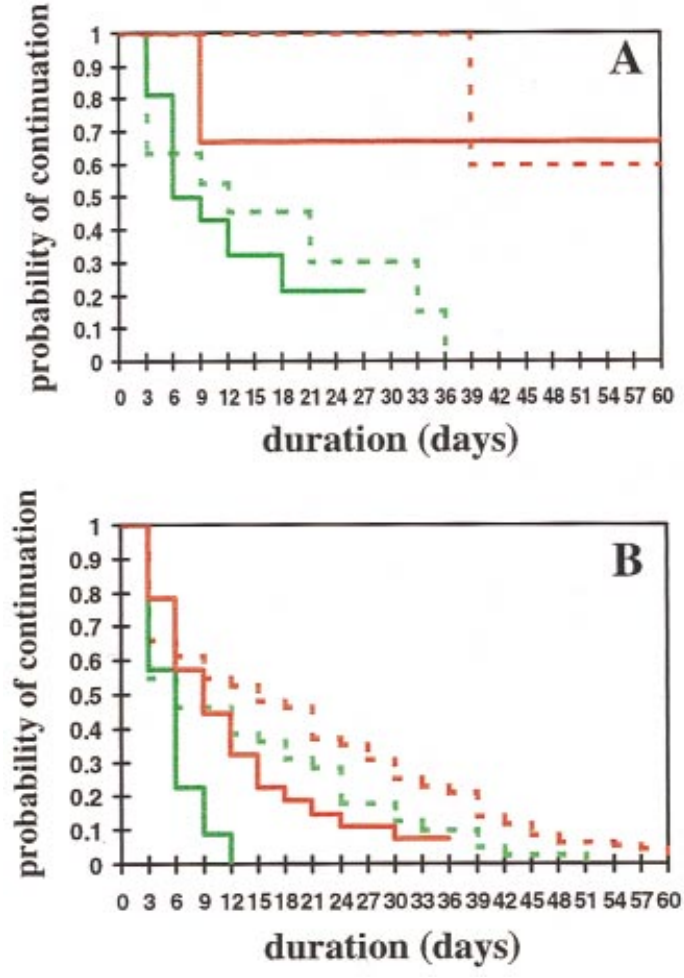

Fig. 5. Kaplan-Meier plots of duration of infection for children aged (A) 4 years and (B) 5-14 years with genotypes of Plasmodium falciparum (red) and $P$. vivax (green). Moderate estimates are solid lines and maximum estimates are broken lines.

and 5-14 years are shown as Kaplan-Meier survival plots (Fig. 5) and the median duration of infection for each estimate is shown in Table 4.

The duration of $P$. falciparum genotypes in 4year-olds was significantly longer than those of the 5 to 14-year-olds when either estimate was used (moderate, $\chi^{2}=8 \cdot 7,2$ D.F., $P=0.012$; maximum, $\chi^{2}=9 \cdot 5,2$ D.F., $\left.P=0.009\right)$. Median duration in 4year-olds exceeded 60 days using both methods (Fig. $5 \mathrm{~A}$ and Table 4). In 5 to 14 -year-olds $P$. falciparum maximum estimates were significantly longer than the moderate ones $\left(\chi^{2}=6.9,1\right.$ D.F., $\left.P=0.009\right)$.

Moderate $P$. vivax duration estimates from the 4year-old age group were also significantly longer than those of 5 to 14 -year-olds $\left(\chi^{2}=10 \cdot 5,2\right.$ D.F., $P=0 \cdot 015)$. Significance was abolished when the maximal estimates were tested $\left(\chi^{2}=1 \cdot 6,2\right.$ D.F., $P=$ 0.446). The duration of P. falciparum and $P$. vivax genotypes cannot be directly compared due to the 10 -fold difference in the sensitivity level of the 2 genotyping techniques. Comparison of estimates of episode duration using blood smear data and the moderate genotype method revealed no significant differences for the same age groups for either $P$. falciparum or $P$. vivax ( $P$. falciparum: 4 years, $\chi^{2}=$ 0.3, 1 D.F., $P=0 \cdot 579$; 5-14 years, $\chi^{2}=3 \cdot 3$, 1 D.F., $P=0.071 ; P$. vivax: 4 years, $\chi^{2}=0 \cdot 6,1$ D.F., $P=$ 0.455 ; 5-14 years, $\chi^{2}=0.4,1$ D.F., $\left.P=0.551\right)$.
Relating multi-species genotype detection and parasite density data

Plotting the dynamics of genotypes of both $P$. falciparum and $P$. vivax in individual children gave a fuller picture of the co-infections within multiply infected children. By also plotting total and speciesspecific parasite density obtained from microscopy and relating this to the genotypes of $P$. falciparum and $P$. vivax present at each point, detailed pictures of how the dynamics of multiple infections were related within each child was built up. If multiple genotypes are present in a sample where the species density is known the density of individual genotypes cannot be determined due to the non-quantitative nature of the genotyping methods. The greater sensitivity of the genotyping methods often allowed determination of genotype dynamics below the microscopy detection level. The dynamics of parasite density together with detection data of individual genotypes of $P$. falciparum and $P$. vivax are shown for 6 representative children (Fig. 6).

The long duration of $P$. falciparum genotypes observed in 4-year-old children is illustrated in Fig. $6 \mathrm{~A}$ and B. P. falciparum has the greatest density over the longest time in child 5 (Fig. 6A) whilst P. vivax was seen as short, intermittent episodes of much lower density. Despite this the complexity of $P$. vivax infection was much greater than of $P$. falciparum.

A greater number of $P$. vivax compared with $P$. falciparum genotypes was also observed in another 4year-old, child 1 (Fig. 6B). This child harboured a single $P$. falciparum infection continuing over 57 days but the parasite density of this genotype changes by more than 2 orders of magnitude during this time. $P$. falciparum was not detected by microscopy in the latter part of the study when infection was dominated by $P$. vivax. During this period PCR genotyping revealed that the same $P$. falciparum infection was maintained at low density throughout the $P$. vivax episode. In contrast to $P$. falciparum, the $P$. vivax episode was the result of infection with more than 1 genotype with variable durations.

$P$. falciparum dynamics resembled more those of $P$. vivax in children older than 4 years, being more complex and of shorter duration. The greatest number of $P$. falciparum genotypes, 9, was detected in child 31, aged 10 years (Fig. 6C). Recurrent behaviour was seen in P. falciparum genotypes 13 and 14. They were detected at the beginning of the study period (from days $0-6$ and 3-18, respectively) and again towards the end (day 57 and days 39 and 42 , respectively). $P$. vivax infection was not as complex as $P$. falciparum in this child, mirroring the paucity of $P$. vivax smear-positive samples.

The numbers of different $P$. falciparum and $P$. vivax genotypes were more equitable in other 
Table 4. Median duration (in days) of Plasmodium falciparum and $P$. vivax genotype episodes by age group, estimated using the moderate and maximum methods

(Median duration is obtained from Kaplan-Meier plots (Fig. 5); $95 \%$ confidence intervals in parentheses.)

\begin{tabular}{lll}
\hline \hline & 4 years & $5-14$ years \\
\hline P. falciparum moderate & $>60$ & 9 \\
& $(9->60)$ & $(6-12)$ \\
P. falciparum maximum & $>60$ & 15 \\
& $(39->60)$ & $(9-21)$ \\
P. vivax moderate & 6 & 6 \\
& $(6-18)$ & $(3-6)$ \\
P. vivax maximum & 12 & 6 \\
& $(3-33)$ & $(3-15)$ \\
\hline \hline
\end{tabular}

children, e.g. child 22 (Fig. 6D). Here the duration and density associated with the $4 P$. falciparum genotypes was variable. $P$. falciparum genotype 4 was present from day 15 , was seen at consecutive points from day 27 to 42 and recurs on day 51 . During this time a second $P$. falciparum infection was detected (genotype 16). This genotype was present from day 30 to 39 . These dynamics show that the immune response(s) responsible for clearing genotype 16 were not acting on genotype 4 , which continues throughout the genotype 16 infection.

The 6 day periodicity of detection of $P$. falciparum genotypes is illustrated in data from child 33 , aged 10 (Fig. 6E). P. falciparum genotype 5 was detected on days 9 and 15. It was out of phase with genotype 4 , which was present on days 12,18 and 24. This genotype was not detected on day 30 but remains in phase with the first episode when it recurred on days 36 and 42. During this period there was substantial variation in the density associated with this genotype.

$P$. falciparum and $P$. vivax infection detectable by microscopy was reduced both in the number of time points and density in child 10, aged 8 (Fig. 6F). Despite this, multiple genotypes of each species were detected in this child but the pattern of detection was more sporadic. Even in this case, the same genotypes were maintained for a substantial period of time.

\section{DISCUSSION}

This study presents for the first time an analysis of the dynamics of co-infections of multiple genotypes from multiple species of Plasmodium in naturally infected children. The diversity and dynamics of individual infections of both P. falciparum and $P$. vivax are highly complex and vary considerably between but also within children. Despite this, some generalizations can be made which provide insights into the burden of multiple asymptomatic malaria infections and how these are regulated by host immune reactions in individuals with some degree of naturally acquired immunity.

Different infections were distinguished by allelic diversity at species-specific polymorphic loci. The number of alleles distinguishable at the $P$. vivax Msp $3 \alpha$ locus was almost as high as that detected at the P. falciparum Msp2 locus despite the reduced sensitivity of the P. vivax PCR amplification. This is because of RFLP determination used in the analysis of Msp3 $\alpha$ detects point mutations whereas the Msp2 method relies upon detection of major size polymorphisms. Size polymorphism was also observed in the Msp3 $\alpha$ gene but the extent of this may be limited by functional constraints on this molecule (Galinski et al. 1999).

The mean multiplicity of $P$. falciparum infections per sample was less than in other highly endemic regions (Babiker, Ranford-Cartwright \& Walliker, 1999) but was similar to results of other surveys in the Madang area of PNG (Paul et al. 1995). P. vivax multiplicity per sample was similar to that estimated in another study in PNG (Kolakovich et al. 1996) and one from India (Joshi et al. 1997) but major differences in sample collection and genotyping techniques makes such comparisons of limited epidemiological value. Similarly, the mean multiplicity of infection of $P$. falciparum and $P$. vivax in this study cannot be compared because of the difference in PCR sensitivity ( $P$. vivax, 100 parasites $/ \mu 1$ (Bruce et al. 1999), P. falciparum 10 parasites $/ \mu 1$ ) in the genotyping method and possible differences in the actual allelic diversity at each locus. The absence of correlation between either $P$. vivax or $P$. falciparum multiplicity per sample and species-specific parasite density in this data set is similar to results from children aged 3-7 years in Tanzania (Smith et al. 1999a) and is in correspondence with the acquisition of density-dependent regulation of parasitaemia by the age of 4 years in PNG (Bruce et al. 2000a).

Although mean multiplicity values per sample are often calculated from longitudinal data, such statistics are biased by the temporal correlation structure within multiple samples. A more useful statistic is the number of infections per child during the study. This gives a better estimate of the burden of infection. All but 1 child had multiple infections as discerned by either species- or molecular typing. Multiple $P$. vivax infections were commonplace in children of all ages. The reduction in the complexity of $P$. falciparum infections in 4-year-old children compared with older children is in line with the agedependency of the complexity of this species in Tanzania (Smith et al. 1999a).

Periodicity in the detection of $P$. falciparum genotypes is likely to be a result of the synchronous replication and the sequestration characteristics of this species in combination with the 3 day sampling 

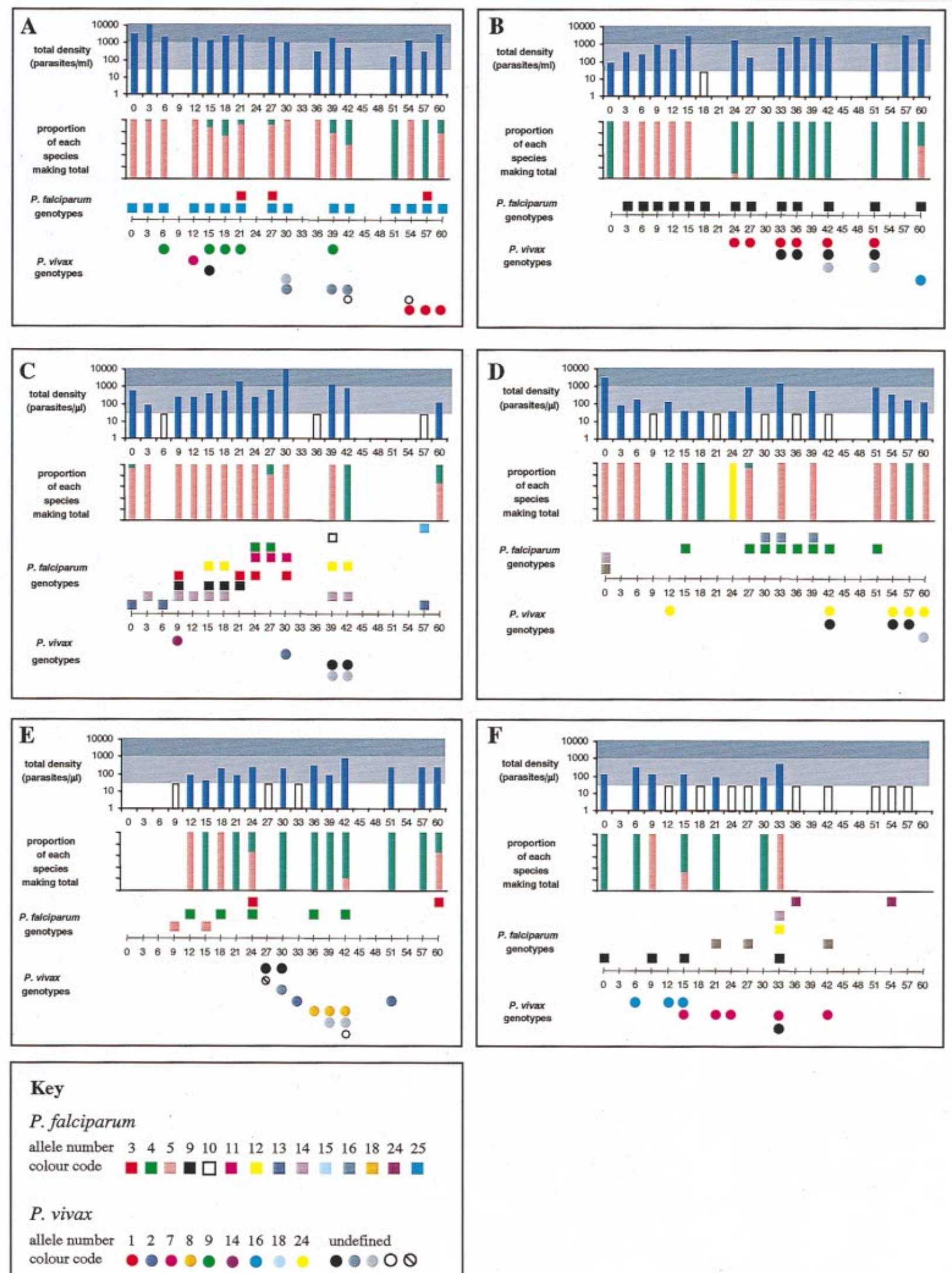

Fig. 6. Dynamics of Plasmodium falciparum and $P$. vivax genotypes in relation to parasite density are shown for (A) child 5; (B) child 1; (C) child 31; (D) child 22; (E) child 33 and (F) child 10. Bars on upper graphs (blue) show total parasite density on a log scale. The proportion of each species is shown in the second graph, P. falciparum, red; $P$. vivax, green; P. malariae, yellow. Light grey shading indicates density between the microscopy sensitivity level (40 parasites $/ \mu 1)$ and the approximate fever threshold (1000 parasites/ $\mu$ l (Cox et al. 1994)). Dark grey shading shows 
protocol. Daily periodicity in $P$. falciparum genotype detection has been previously demonstrated in asymptomatic patients in Tanzania (Färnert et al. 1997). Statistical analysis of the periodicity in this study provides quantitative evidence that single genotypes within individuals show a tendency for synchronous replication. The periodicity of individual genotypes underlies the periodicity detected by microscopy in the same children (Bruce et al. $2000 \mathrm{~b}$ ). The absence of synchrony amongst multiple co-infecting $P$. falciparum genotypes suggests that the maintenance of periodicity is governed mainly by alignment of the parasite replication cycle with host diurnal rhythms (Coatney \& Stubbs, 1940; Hawking, Worms \& Gammage, 1968) rather than killing of parasites by fever, which would synchronize the cycles of all genotypes present (Kwiatkowski \& Greenwood, 1989). Epidemiological studies of $P$. falciparum must take the tendency for synchronous replication into account in the design of sampling protocols. This is especially important when detection of individual genotypes is used as an end point measure in longitudinal drug or vaccine trials.

The observed dynamics of infection showed that host immunity which results in reduction of parasite density to below PCR detectable levels, was acting against genotypes of a single species independently and that clearance of one genotype was not linked with clearance of others. This is in line with observations that show that the immunity that clears parasites in humans is predominantly species- and genotype-specific (Taliaferro, 1939; Jarra \& Brown, 1985).

Episodes of each species have been shown to follow a sequential rather than concurrent pattern in this data set (Bruce et al. 2000a). The sequential pattern of infection is statistically different to that expected by chance alone and total parasite density has been shown to follow density-dependent dynamics in highly parasitized children (Bruce et al. $2000 a$ ). Density-dependent regulation, which is species- and genotype-transcending in nature, is thought to play a role in the regulation of parasitaemia and be responsible for interactions between different species. To be able to detect interactions between different genotypes of a single species the density of each genotype is required. The non-quantitative genotyping methods used in this study preclude any conclusions about genotype interactions.

The dynamics of parasitaemia from blood smears in combination with PCR genotyping in children with single $P$. falciparum genotypes show the occurrence of major changes in the density of a single genotype over short periods. Such changes may correspond with specific clearance of parasites expressing a particular RBC surface antigenic variant. PCR detection has demonstrated the persistence of the same genotype below microscopy detection levels following such clearance, during the rise of parasitaemia of another species. Survival of a minority of parasites expressing a different antigenic type from the majority of the same population can explain these dynamics. These data are the first to show that dynamics consistent with antigenic variation (Reeder \& Brown, 1996) occur in naturally infected humans carrying multiple Plasmodium infections.

Two measures of the duration of infection of genotypes termed moderate and maximum were made. Moderate estimates used the same rules as those from blood smears (Bruce et al. 2000b) and provide a measure of individual episodes of infection. Using this measure, episodes of $P$. falciparum and $P$. vivax were longer in younger children. Decreasing duration of episodes of individual genotypes underlies the similar trend with age for both species in microscopy data (Bruce et al. 2000b). Selection of the most parasitized children for genotyping may have biased genotype durations towards slightly longer estimates but the comparison of relative duration across age groups remains valid. $P$. falciparum episodes showed the greatest difference in duration, between 4-year-old and 5 to 14-year-olds. This longer duration explains the absence of a correlation of the number of $P$. falciparum genotypes with the proportion of smear positive samples in 4year-olds (Bruce et al. 2000 a).

The reduction of the duration of infection of individual genotypes with age demonstrated here is in agreement with data from children of similar ages in Tanzania, where the duration of infection (calculated as the reciprocal of the recovery rate) decreases slightly in older children and more significantly into adulthood (Smith et al. 1999a) although the authors did not interpret their data in this way (Smith et al. $1999 b)$.

Surprisingly, moderate estimates of episode duration of individual genotypes did not vary significantly from those made from blood smear results for either species and all age groups. The greater sensitivity of $P$. falciparum genotyping compared to microscopy was expected to result in longer estimates for this parasite from genotyping. The small number

parasite density above the fever threshold. Open bars, represent smears which were negative by microscopy. Where bars are missing samples were absent. Individual genotypes of $P$. falciparum are shown as squares and $P$. vivax genotypes as circles. Different colours represent genotypes defined by alleles of Msp2 and Msp3 $\alpha$ as in the key. $P$. vivax genotypes shown in black or grey are undefined. 
of infections analysed may have been insufficient to allow detection of differences using these two methods. The similarity of episode duration estimated from microscopy and genotyping may not hold for areas of higher endemicity where a greater percentage of children will be constantly smearpositive.

The duration of episodes of infection with $P$. falciparum and $P$. vivax genotypes cannot be directly compared because of the difference in sensitivity of the PCR genotyping. Microscopy does not have the same limitation because the sensitivity of detection of both $P$. falciparum and $P$. vivax infections is equal. $P$. vivax infections measured by microscopy tended to be shorter in duration than P. falciparum infections in all age groups (Bruce et al. 2000 b). This is likely to be a result of a real difference in the duration of $P$. vivax genotypes. This difference could be explained by faster acquisition of specific immunity to $P$. vivax. Supporting evidence for this comes from induced infection experiments in which fewer inoculations were required to produce immunity to homologous $P$. vivax than to $P$. falciparum infections (discussed by Taliaferro, 1939). It has also been suggested that faster acquisition of immunity to $P$. vivax explains the quicker decline in age prevalence curves for this species in regions where both species exist (Maitland et al. 1996; Maitland, Williams \& Newbold, 1997).

Maximum estimates of genotype duration accounted for recurrence in the detection of genotypes. Recurrence of the same genotype can be due to either recrudescence of the same infection or to reinfection with a different infection that carries the same allele. The probability of re-infection with the same genotype can be approximated from the sum of the squares of genotype frequency per child (0.060 for P. falciparum and 0.064 for $P$. vivax calculated from Tables 2 and 3, respectively). This assumes that (1) the frequency of genotypes in blood is equal to that transmitted from mosquitoes, (2) the probability of re-infection is equal between individuals and (3) infection with a genotype is a random event. These assumptions may not hold and therefore, these values should be viewed only as rough estimates. They do, however, suggest that recrudescence rather than re-infection is more likely to explain the majority of recurrences. In children with multiple infections, recurrence may also be due to the limitation of the PCR genotyping techniques that can fail to detect a genotype if others are present at a ratio greater than 1:10 (unpublished observations).

The maximum measure is a better estimate of duration of genotypes than the moderate estimate as it accounts both for recrudescence and possible PCR anomalies that prevent detection of genotypes in multiply infected samples. However, the total duration of infection with each genotype may be much longer than the 61 day duration of this study (James, Nicol \& Shute, 1932; Roper et al. 1998). It has been suggested that increased multiplicity of $P$. falciparum in older children is the result of the cumulated chronicity of multiple infections over long periods of time (Smith et al. 1999b). Recurring chronic infections that become undetectable between episodes cannot be distinguished from newly inoculated parasites when transmission is ongoing. Similarly, relapses from dormant liver stages of $P$. vivax cannot be distinguished from new infections. Measurement of the total duration of malaria infections therefore poses a significant challenge in endemic regions where the high rate of multiple infections prevents the use of multi-locus genotyping to more accurately distinguish parasite populations detected at different times.

We would like to thank the people of Gonoa for their longstanding co-operation and for their tolerance throughout the study period. Our thanks also to the staff of the Papua New Guinea Institute of Medical Research at Madang for their assistance, and especially to the members of the field team who assisted with sample collection. This work is dedicated to the memory of Helena Vrbova and Nicky Gibson, whose contribution to malaria research in PNG was substantial. Field work was funded by a grant from The European Commission, STD 3, to D. W., K. P. D. and M. P. A. K. D. and C. A. D. are funded by The Wellcome Trust. M. B. was funded by a studentship from The British Medical Research Council and by The Wellcome Trust. The research conducted here was also supported by National Institutes of Health (NIH) grant AI24710 awarded to J. W. B., and NIH grant AI37545 and WHO/TDR grants (950440 and 910495) awarded to M. R. G.

\section{REFERENCES}

AL-KHEDERY, B., BARNWELL, J. W. \& GALINSKI, M. R. (1999). Antigenic variation in malaria: A $3^{\prime}$ genomic alteration associated with the expression of a $P$. knowlesi variant antigen. Molecular Cell 3, 131-141. BABIKER, H. A., RANFORD-CARTWRIGHT, L. C., CURRIE, D., CHARLWOOD, J. D., BILlingsley, P., TEUSCHER, T. \& WALLIKER, D. (1994). Random mating in a natural population of the malaria parasite Plasmodium falciparum. Parasitology 109, 413-421.

BABIKER, H. A., RANFORD-CARTWRIGHT, L. C. \& WALLIKER, D. (1999). The epidemiology of multiple Plasmodium falciparum infections 3. Genetic structure of Plasmodium falciparum infections in the Kilombero region of Tanzania. Transactions of the Royal Society of Tropical Medicine and Hygiene 93 (Suppl. 1), $\mathrm{S} 1 / 11-\mathrm{S} 1 / 14$.

BARUCH, D. I., PASlOSKe, B. L., SINGH, H. B., BI, X., MA, X. C., FEldman, M., TARASChi, T. F. \& HOWARD, R. J. (1995). Cloning the Plasmodium falciparum gene encoding PfEMP1, a malarial variant antigen and adherence receptor on the surface of parasitized human erythrocytes. Cell 82, 77-87.

BJÖRKMAN, A., DO ROSARIO, v. E., SNOUNOU, G. \& WAlliker, D. (1998). Standardizing PCR for molecular epidemiology studies of malaria. Parasitology Today 14, 85. 
BROWN, K. N. \& BROWN, I. N. (1965). Immunity to malaria: antigenic variation in chronic infections of Plasmodium knowlesi. Nature, London 208, 1286-1288. BRUCE, M. C., DONNELly, C. A., ALPERS, M. P., GALINSKI, M. R., BARNWELL, J. W., WALLIKER, D. \& DAY, K. P. $(2000 a)$. Cross-species interactions between malaria parasites in humans. Science 287, 845-848.

BRUCE, M. C., DONNELly, C. A., PACKer, M., LAGOG, M., GIBSON, N., NARARA, A., WALliker, D., ALPERS, M. P. \& DAY, K. P. $(2000 b)$. Age- and species-specific duration of infection in asymptomatic malaria infections in Papua New Guinea. Parasitology 121, 247-256. BRUCE, M. C., Galinski, M. R., BarnWell, J. W., SNOUOU, G. \& DAY, K. P. (1999). Polymorphism at the Msp3 $\alpha$ locus of $P$. vivax: global and local diversity. American Fournal of Tropical Medicine and Hygiene 61, 518-525. CiUCA, M., Ballif, L. \& Chelarescu-vieru, M. (1934). Immunity in malaria. Transactions of the Royal Society of Tropical Medicine and Hygiene 27, 619-622. COX, M. J., KUM, D., TAVUl, L., NARARa, A., RaIKo, A., Alpers, M., Medley, G. \& DAY, K. P. (1994). Dynamics of malaria parasitaemia associated with febrile illness in children from a rural area of Madang, Papua New Guinea. Transactions of the Royal Society of Tropical Medicine and Hygiene 88, 191-197.

Daubersies, P., Sallenave sales, s., Magne, S., Trape, J. F., CONTAMin, H., FAndeur, T., Rogier, C., MERCEREAU-PUIJALON, O. \& DRUILHE, P. (1996). Rapid turnover of Plasmodium falciparum populations in asymptomatic individuals living in a high transmission area. American Fournal of Tropical Medicine and Hygiene 54, 18-26.

FÄRNERT, A., SNOUNOU, G., ROOTH, I. \& BJÖRKMAN, A. (1997). Daily dynamics of Plasmodium falciparum subpopulations in asymptomatic children in a holoendemic area. American Fournal of Tropical Medicine and Hygiene 56, 538-547.

FENTON, B., CLARK, J. T., ANJAM KHAN, C. M., ROBINSON, J., WALliker, D., RIDLEY, R. G., SCAIFFE, J. G. \& McBride, J. S. (1991). Structural and antigenic polymorphism of the 35- to 48- kilodalton merozoite surface antigen (MSA-2) of the malaria parasite Plasmodium falciparum. Molecular and Biochemical Parasitology 11, 963-971.

GALINSKI, M. R., CORREDOR-MEdiNA, C., POVOA, M., Crosby, J., ingravallo, P. \& Barnwell, J. W. (1999). Plasmodium vivax merozoite surface protein- $3 \alpha$ contains coiled-coil motifs within an alanine-rich central domain. Molecular and Biochemical Parasitology 101, 131-147.

HAWking, F., WORMS, M. J. \& Gammage, K. (1968). 24 and 48-hour cycles of malaria parasites in the blood; their purpose, production and control. Transactions of the Royal Society of Tropical Medicine and Hygiene 62, 731-760.

JAMES, S. P., NiCOL, W. D. \& SHUte, P. G. (1932). A study of induced malignant tertian malaria. Proceedings of the Royal Society of Medicine 25, 1153-1186.

JARRA, W. \& BROWN, K. N. (1985). Protective immunity to malaria: studies with cloned lines of Plasmodium chabaudi and P. bergei in CBA/Ca mice. I. The effectiveness and inter- and intra-species specificity of immunity induced by infection. Parasite Immunology 7, 595-606.
Joshi, H., SUbBaraO, S. K., NANDA, N., GHOSH, S. K., CARTER, R. \& SHARMA, v. P. (1997). Genetic structure of Plasmodium vivax isolates in India. Transactions of the Royal Society of Tropical Medicine and Hygiene $\mathbf{9 1}$, 231-235.

KOlakovich, K. A., SSENGoba, A., WOJCiK, K., TSUBoi, T., AL-YAMAN, F., ALPERS, M. P. \& ADAMS, J. H. (1996).

Plasmodium vivax: favoured gene frequencies of the merozoite surface protein-1 and the multiplicity of infection in a malaria endemic region. Experimental Parasitology 83, 11-18.

KWIATKowski, D. (1995). Malarial toxins and the regulation of parasite density. Parasitology Today 11, 206-212.

KWIATKOWSKI, D. \& GREENWOOD, B. M. (1989). Why is malaria fever periodic? A hypothesis. Parasitology Today 5, 264-266.

KWIATKOWSKI, D. \& NOWAK, M. (1991). Periodic and chaotic host-parasite interactions in human malaria. Proceedings of the National Academy of Sciences, USA 88, 5111-5113.

KYES, S., CRAiG, A. G., MARSH, K. \& NEWBOLD, C. I. (1993). Plasmodium falciparum: a method for the amplification of $\mathrm{S}$ antigens and its application to laboratory and field samples. Experimental Parasitology 77, 473-483.

Maitland, K., Williams, T. N., BENNETT, S., NEWBold, C. I., PETO, T. E. A., Viji, J., TIMOThY, R., CleGG, J. B., Weatherall, D. J. \& BOWDEN, D. K. (1996). The interaction between $P$. falciparum and $P$. vivax in children on Espiritu Santo, Vanuatu, South West Pacific. Transactions of the Royal Society of Tropical Medicine and Hygiene 90, 614-620.

Maitland, K., Williams, T. N. \& NEWBold, C. I. (1997). Plasmodium vivax and P. falciparum: Biological interactions and the possibility of cross-species immunity. Parasitology Today 13, 227-231.

MENDIS, K. N., IHALAMUlLa, R. I. \& DAVID, P. H. (1988). Diversity of Plasmodium vivax-induced antigens on the surface of infected human erythrocytes. American Fournal of Tropical Medicine and Hygiene 38, 42-46.

PaUl, R. E. L., PACKer, M. J., Walmsley, M., LaGoG, M., RANFORD-CARTWRIGHT, L. C., PARU, R. \& DAY, K. P. (1995). Mating patterns in malaria parasite populations of Papua New Guinea. Science 269, 1709-1711.

RANFORD-CARTWRIGHT, L. C., BALFE, P., CARTER, R. \& WALliker, D. (1993). Frequency of cross-fertilization in the human malaria parasite Plasmodium falciparum. Parasitology 107, 11-18.

REEDER, J. C. \& BROWN, G. v. (1996). Antigenic variation and immune evasion in Plasmodium falciparum malaria. Immunology and Cell Biology 74, 546-554.

RILEY, E. M., ALLEN, S. J., WEELER, J. G., BLACKMAN, M. J., BENNETT, S., TAKACS, B., SCHONFELD, H.-J., HOLDER, A. \& GREENWOOD, B. M. (1992). Naturally acquired cellular and humoral responses to the major merozoite surface antigen (Pf MSP1) of Plasmodium falciparum are associated with reduced malaria morbidity.

Parasite Immunology 14, 321-337.

roper, C., Richardson, W., Elgassab, I. M., GiHa, H., HVIID, L., SATTI, G. M. H., THEANDER, T. G. \& ARNOT, D. E. (1998). Seasonal changes in the Plasmodium falciparum population in individuals and their 
relationship to clinical malaria: a longitudinal study in a Sudanese village. Parasitology 116, 501-510.

SMith, T., BECK, H.-P., KITUA, A., MWANKUSYe, S., FELGER, I., FRASER-HURT, N., IRION, A., ALONSO, P., TEUSCHER, T. \& TANNER, M. $(1999 a)$. Epidemiology of multiple Plasmodium falciparum infections. 4. Age dependence of the multiplicity of Plasmodium falciparum infections and of other malariological indices in an area of high endemicity. Transactions of the Royal Society of Tropical Medicine and Hygiene 93, (Supp. 1), $\mathrm{S} 1 / 15-\mathrm{S} 1 / 20$.

SMith, T., FELGER, I., TANNER, M. \& BECK, H.-P. (1999 b). The epidemiology of multiple Plasmodium falciparum infections. 11. Premunition in Plasmodium falciparum infection: insights from the epidemiology of multiple infections. Transactions of the Royal Society of Tropical Medicine and Hygiene 93, (Supp. 1), $\mathrm{S} 1 / 59-\mathrm{S} 1 / 64$.
SMYTHE, J. A., COPPEL, R. L., DAY, K. P., MARTIN, R. K., ODUOLA, A. M. J., KEMP, D. J. \& ANDERS, R. F. (1991). Structural diversity in the Plasmodium falciparum merozoite surface antigen 2. Proceedings of the National Academy of Sciences USA 88, 1751-1755. TALIAfERro, W. H. (1939). Immunity to the malaria infections. In Malariology (ed. Boyd, M. F.), pp. 935-965, W. B. Saunders \& Co., Philadelphia.

TAYlor, R. R., ALLEN, S. J., GREENWOOD, B. M. \& RILEy, E. M. (1998). IgG3 antibodies to Plasmodium falciparum merozoite surface protein 2 (MSP2): increasing prevalence with age and association with clinical immunity to malaria. American Fournal of Tropical Medicine and Hygiene 58, 406-413.

young, M. D., COATNEY, G. R. \& STUBBS, T. H. (1940). Studies on induced quartan malaria in Negro paretics. II. The effect of modifying the external conditions. American Fournal of Hygiene 32, 63-70. 